\title{
Java Advanced Imaging API: A Tutorial
}

\author{
Rafael Santos 1
}

\begin{abstract}
This tutorial shows how the Java language and its Java Advanced Imaging (JAI) Application Program Interface (API) can be used to create applications for image representation, processing and visualization. The Java language advantages are its low cost, licensing independence and inter-platform portability. The JAI API advantages are its flexibility and variety of image processing operators.

The purpose of this tutorial is to present the basic concepts of the JAI API, including several complete and verified code samples which implements simple image processing and visualization operations. At the end of the tutorial the reader should be able to implement his/her own algorithms using the Java language and the JAI API.
\end{abstract}

Keywords: Image processing, Algorithms, Java, Java Advanced Imaging.

\section{Introduction}

In spite of the existence of several image processing softwares with many image processing functions, tailored for several different uses, there is often the need for implementation of specific algorithms which are not available on those softwares - for example, a user may want to implement his/her own image classification or filtering algorithm or tweak some already implemented algorithm parameters. Some of those softwares allow the development of user-defined modules, often using the same API developed for the software itself. The developer may be able to use those APIs to develop his/hew own routines, but often there is an additional cost or licensing restrictions.

A royalty-free, portable, flexible alternative for the implementation of generic applications is the Java language [1]. For image processing and representation, the JAI (Java Advanced Imaging) API (Application Program Interface) [2] can be used. Although the API is not part of a full-featured image processing software, the existing functions and extension possibilities allied to the low cost and ease of implementation makes this combination an attractive option for image processing algorithms development.

This tutorial will present some concepts on the JAI API and give code samples and short code snippets for image input and output, application of basic operators, image visualization and image data manipulation. The tutorial will not present some details like installation and configuration issues or advanced operations such as network imaging. It is assumed

\footnotetext{
${ }^{1}$ Divisão de Sensoriamento Remoto - Instituto de Estudos Avançados - Centro Técnico Aeroespacial santos@ieee.org
} 
that the reader already have a good knowledge of Java or other modern languages $(\mathrm{C}++$, Delphi) and basic image processing knowledge.

Instructions for installation the JAI libraries and running applications which use the JAI classes can be found in [2,3]. This tutorial assumes that the user will have access to a complete JDK (Java Development Kit) installation (version 1.4 or later) with the JAI API installed (version 1.1.2 or later).

\section{Image data representation}

Image processing algorithms usually require the manipulation of the image data (pixels). In this section the model used by JAI for image data storage and manipulation will be presented, with the corresponding Java/JAI classes.

Images in JAI may be multidimensional (i.e. with several values associated to a single pixel) and may have pixel with either integer or floating point values (altough there are restrictions on the types of images which can be stored in disk). Pixels may be packed in different ways or unpacked in the image data array. Different color models can be used. As one may expect, in order to be able to represent a variety of image data, one must deal with a variety of classes.

Before showing examples of those classes, the basic classes for iamge data representation will be shown. Some of those classes are abstract, concrete subclasses of those behave on more or less the same way:

Planar Image: Basic class for image representation in JAI, allows the representation of images with more flexibility than the Java class BufferedImage. Both BufferedImage and Planar Image uses several different classes for flexible image data representation: its pixels are stored in an instance of Raster which contains an instance of a concrete subclass of DataBuffer, packed accordingly to the rules descriped by an instance of a concrete subclass of SampleModel. An instance of $\mathrm{Pl}$ anarImage also have a ColorModel associated to it, which contains an instance of ColorSpace, which determines how a pixel's value can be translated to color values. Figure 1 shows how those classes are used to compose an instance of PlanarImage.

A Planar Image is read-only, i.e. it may be created and its pixels values may be read in several different ways, but there are no methods that allow the modification of pixels values. $\mathrm{P}$ lanar Images may have the origin of the image in a position different from the coordinate $(0,0)$, or even pixel coordinates with negative values.

TiledImage: A subclass of PlanarImage, which can be used for reading and writing image data. 
RenderedOp: Another subclass of P lanar Image, which represents a node in a rendered imaging chain. A rendered imaging chain is a powerful and interesting concept of JAI which allows the processing of an image to be specified as a series of steps (operators and parameters) which are applied to one or more images.

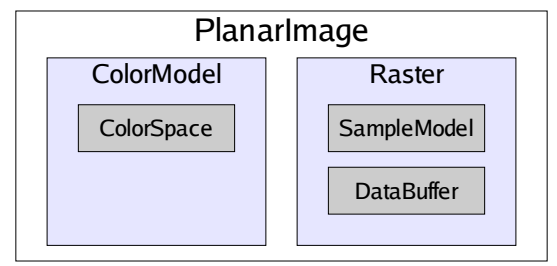

Figure 1. P lanar Image structure (after [3])

Another interesting concept used in JAI are tiled images. Tiles can be considered as subsets of the images which may be processed independently. Large images thus can be processed in Java/JAI with reasonable performance, even through rendered imaging chains, since there is no need to load the whole image data in memory at once. If the image is tiled, all its tiles must have the same width and height. JAI allows different origins for the pixels and for the tiles on an image, although there are few, if any, practical applications for this.

Figure 2 shows a simple tiled image, where the origin of the tiles coincides with the origin of the image but with the tiles extended past the image edges (as it is often the case). When a tile extends past the image edges, its contents are undefined. More information on tiled images may be found in [4].

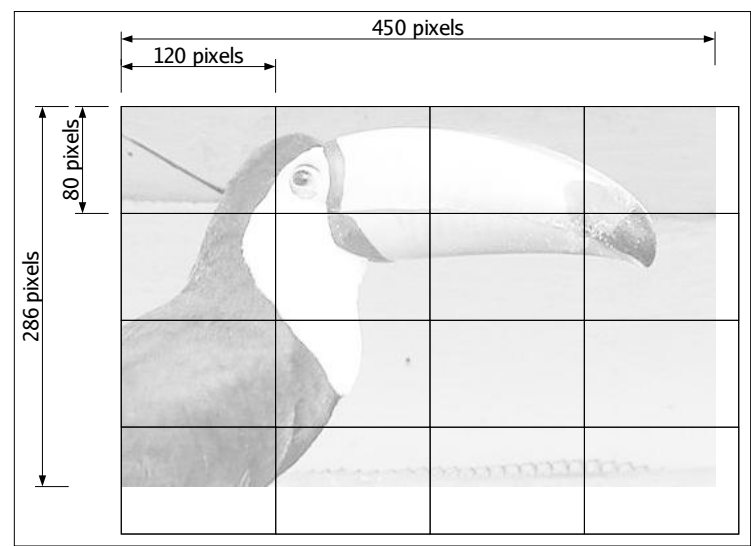

Figure 2. A tiled image. 
With the knowledge of which classes are used for image data representation, it is relatively simple to create an image in-memory for storage or further processing.

Two different examples of creation of images will be presented, the first one will be the creation of a grayscale image with a floating-point pixel data, and the second will be the creation of a RGB image with integer pixel data. Both examples will use the following simple steps:

1. Create the image data in an array in memory. This array must be an unidimensional array, although for simplicity a multidimensional array can be created and flattened later.

2. Create an instance of a concrete subclass of DataBuffer, using one of its constructors and the image data array.

3. Create an instance of SampleModel with the same data type of the DataBuffer and desired dimensions. A factory method of the class RasterFactory may be used for this.

4. Create an instance of ColorModel compatible with the sample model being used. The static method Planar Image. createColorModel may be used for this, using the sample model as an argument.

5. Create an instance of WritableRaster using the sample model and the image data array. The method RasterFactory . createWritableRaster can be used for this.

6. Create a writable image (instance of TiledImage) using the sample model, color model and dimensions.

7. Associate the instance of Raster with the image using the method setData of the class TiledImage.

8. Do something with the instance of $\mathrm{Ti}$ ledImage, like saving it to disk, displaying or processing it.

The code for those steps (as a complete Java application) that will create, as a result, a floating-point one-banded (grayscale) image is shown in listing 1. Due to space constraints, only the essential working code and comments will be shown in this tutorial. The reader may find more complete code for this and other examples, with comments, on [5].

Listing 1: Class CreateGray Image.

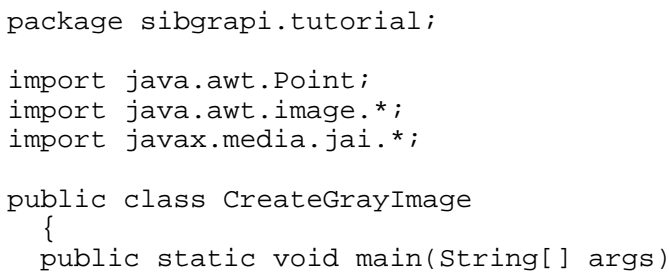




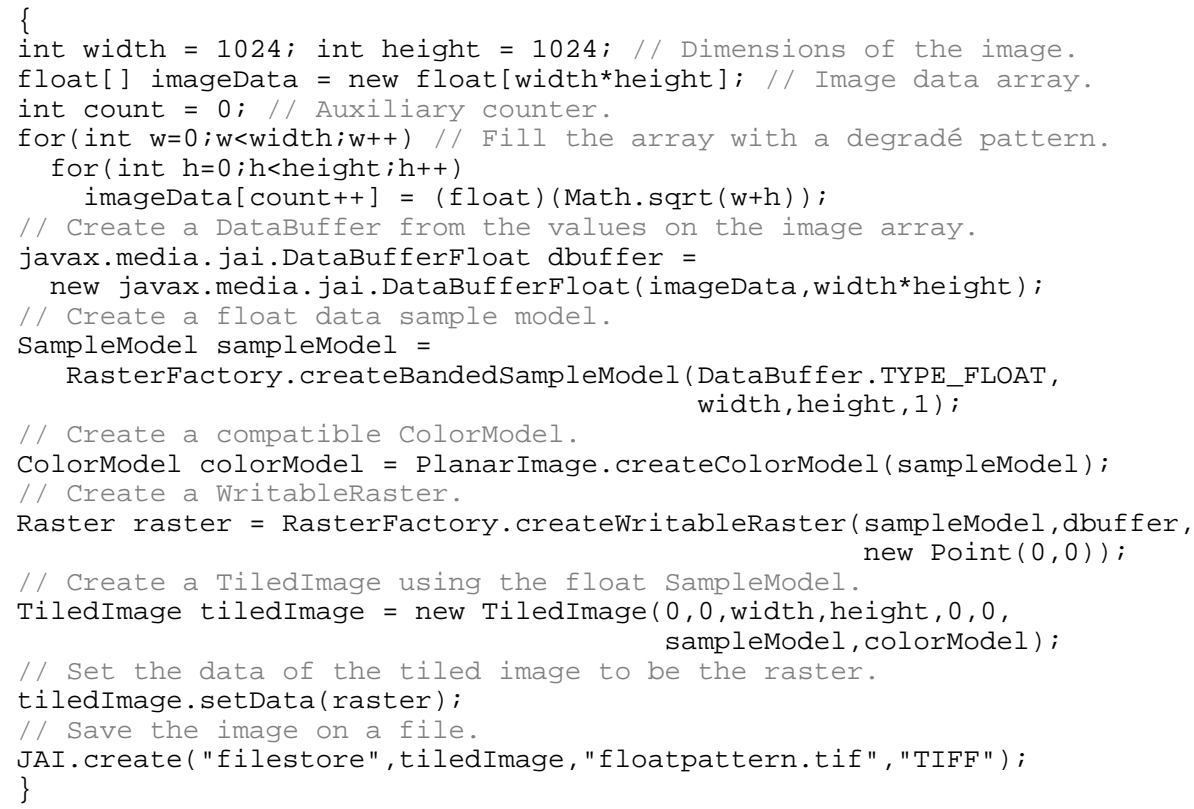

Similar code for creation of a RGB image with a simple red and blue pattern is shown in listing 2. Again, the same basic steps are used, although instances of different concrete classes that inherit from DataBuffer and SampleModel are used.

Listing 2: Class CreateRGBImage.

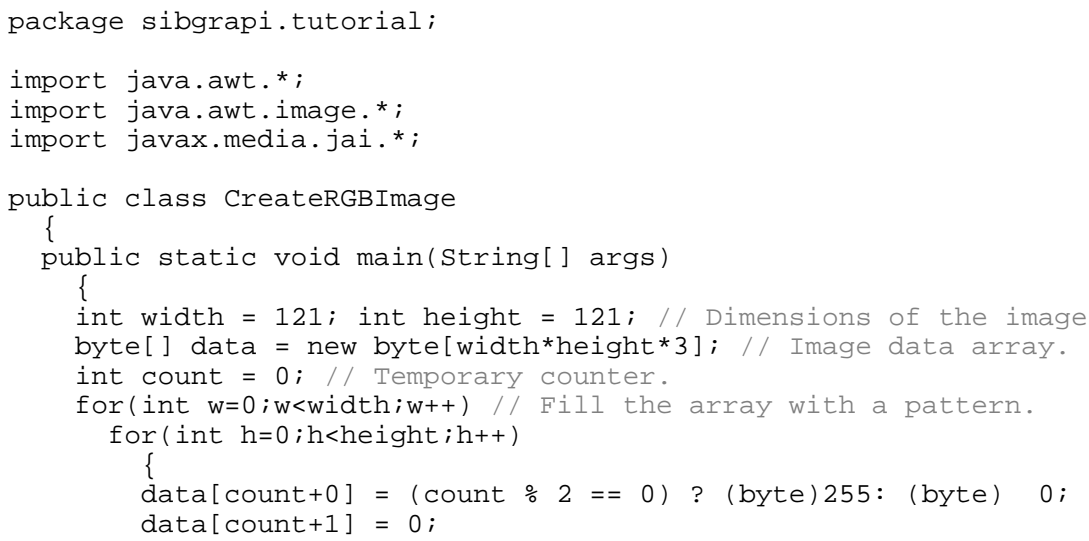




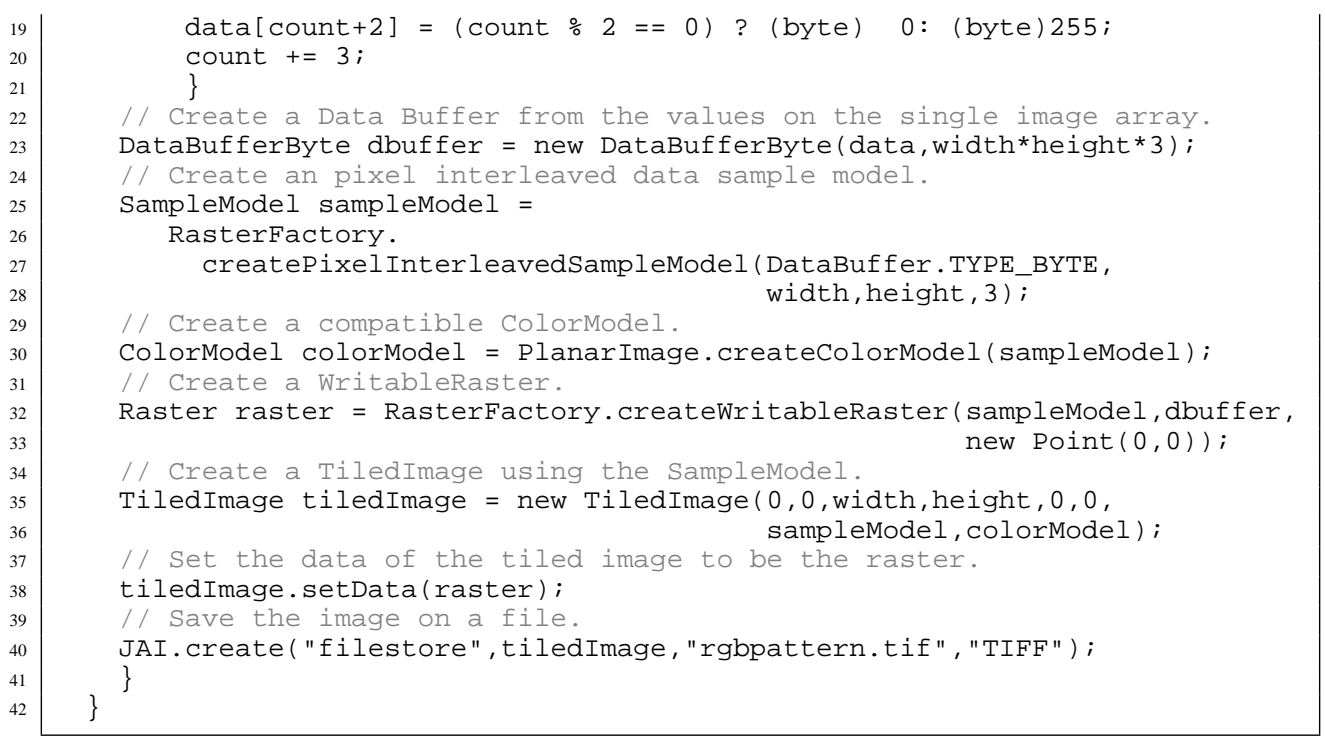

In order to get information about an existing image, several get methods from the classes PlanarImage, SampleModel and ColorModel can be used. Several of those methods are demonstrated in the code on the listing 3, which is a complete Java application which must get, as a command-line parameter, the file name of an existing image.

Listing 3: Class ImageInfo.

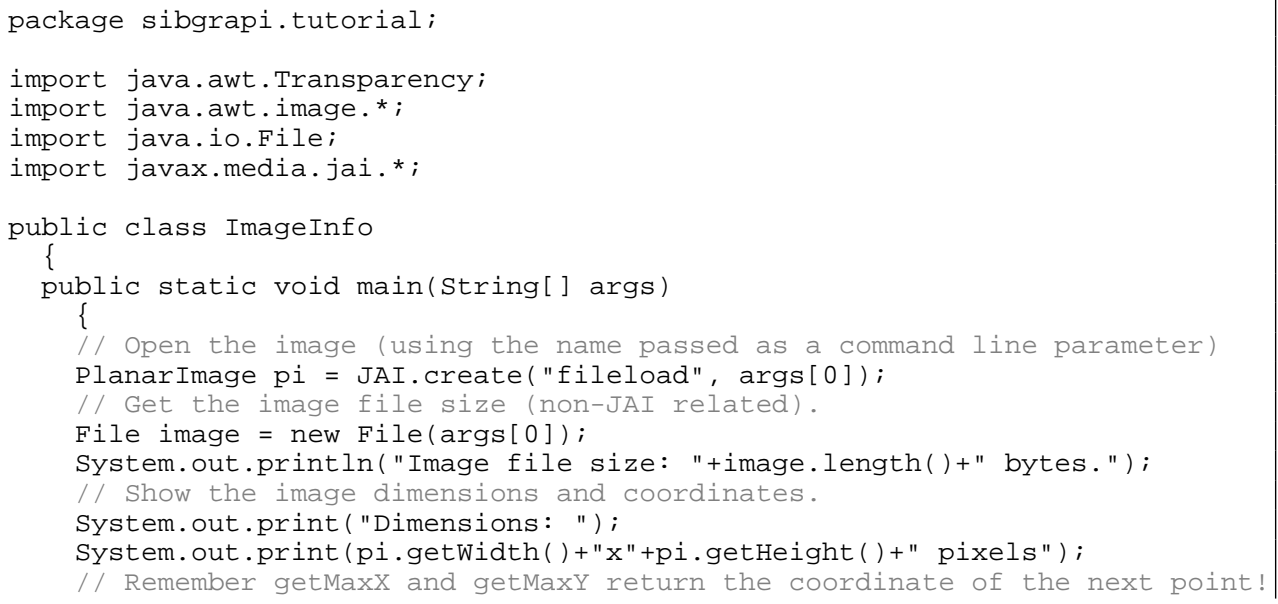




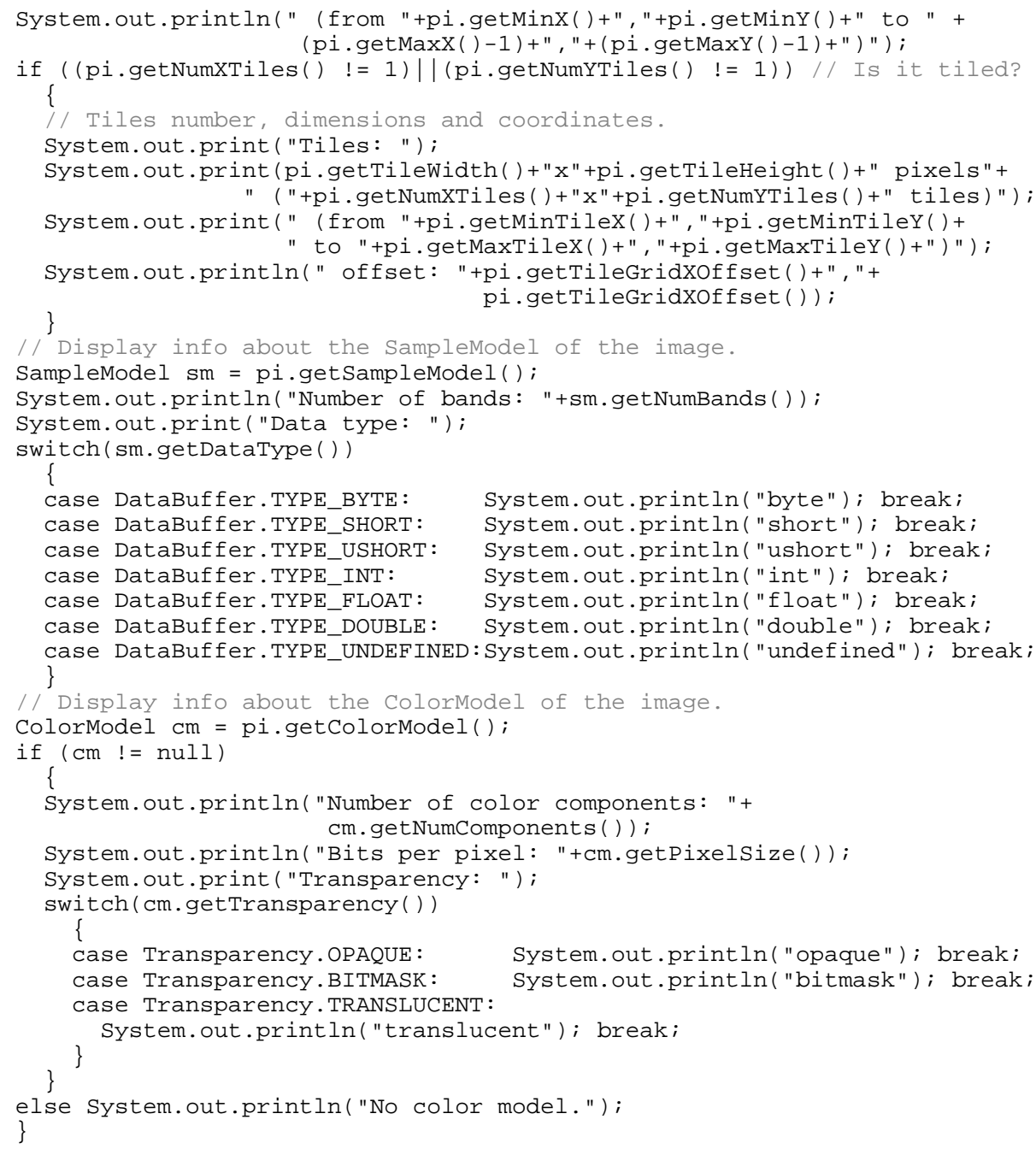

\section{Simple JAI operators}

The JAI API contains several image operators which can be applied with minimum programming. Those operators follow the concept of a rendered imaging chain, where the steps for the image processing are defined but will be carried only when needed (deferred 
execution).

Those operations are specified in a simple way: first an instance of ParameterBlock is created, which is basically a vector of data that will be used for the operation, then the static method create of the class JAI is executed. This method gets as an argument a name for the operation and the instance of ParameterBlock and returns an instance of RenderedOp which can be manipulated as a Planar Image. Alternatively one can add the original image in the instance of ParameterBlock as a parameter to its addSource method. Other parameters are added to the ParameterBlock with its add method. Other forms of the method does not require a ParameterBlock and accept other arguments.

One example of a JAI operator is the "filestore" operator, used in the code in the listings 1 and 2 to store an instance of PlanarImage (or of a subclass of it) in a file. The call for the JAI. create method used as arguments the name of the operator, the instance of P lanar Image, a file name and a string containing the desired image file name ("TIFF", "JPEG", "PNG", etc.).

Another example of operator, which does not use the instance of ParameterBlock was already shown in listing 3: a call to JAI. create ("fileload", imageName); will load and return an image which file name is contained on the string imageName. Other operators and code snippets that illustrate its usage will be shown in this section. A list of all operators can be found on the JAI API documentation [6], on the documentation for the package javax.media.jai.operator.

The "invert" operator requires a simple P lanar Image as input, and can be executed as shown in the code in listing 4, which shows how to read and invert an image.

Listing 4: Code for image inversion.

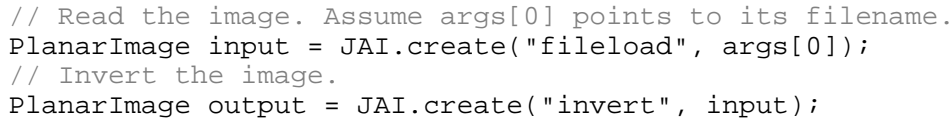

The "scale" operator scales one image giving a scaled version as a result. It optionally may also translate the image. To use this operator, one need to create a Parameter Block and add the original image, two floating point values corresponding to the $\mathrm{X}$ and $\mathrm{Y}$ scale and another two floating point values corresponding to the translation in $\mathrm{X}$ and $\mathrm{Y}$ of the images' pixels. When scaling an image, interpolation of the pixels must be performed, therefore one need also to add to the parameter block an instance of a concrete subclass of javax.media.jai. Interpolation. The code in listing 5 shows one example of usage of this operator.

ParameterBlock $\mathrm{pb}=$ new ParameterBlock (); 
Listing 5: Code for image scaling.

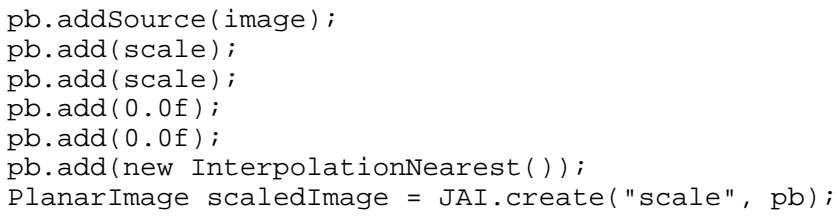

The "rotate" operator rotates one image using an angle in radians. Similarly to the "scale" operator, it also needs an interpolation method. In order to use this operator, one must create a ParameterBlock, add an image source to it, and add (in this order) the rotation angle, the two coordinates for the center of the rotation and an instance of a concrete subclass of Interpolation. The code in listing 6 shows one example of usage of the "rotate" operator, which rotates an image 45 degrees around its center.

Listing 6: Code for image rotation.

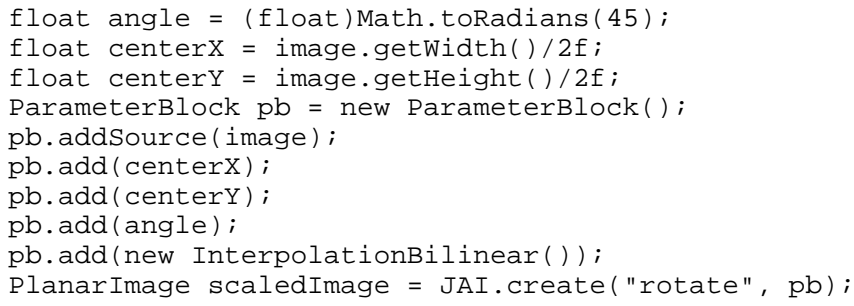

Convolution can be easily done with JAI. The "convolve" operator performs convolution of an image with a kernel, which can be created as an instance of the class Kernel JAI. This instance is created with an array which represents the kernel values, then the instance of KernelJAI may be used even without a ParameterBlock. The code in listing 7 shows how one can create a $15 \times 15$ smoothing kernel and apply it to an input image, giving as a result an output image. The kernel values must be normalized, i.e. they must sum up to one.

Listing 7: Code for image smoothing.

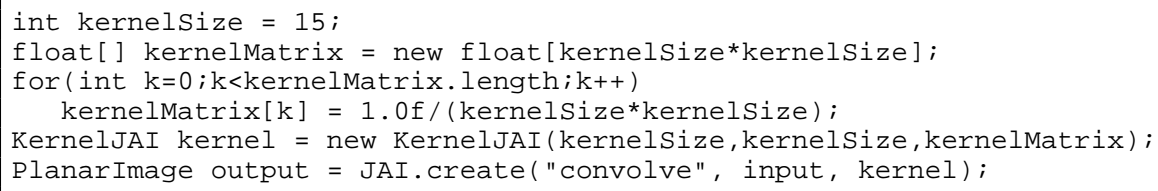


As another example, the code in listing 8 shows how one can create and apply a horizontal Sobel operator to an input image. 
Listing 8: Code for Sobel edge detection in an image.

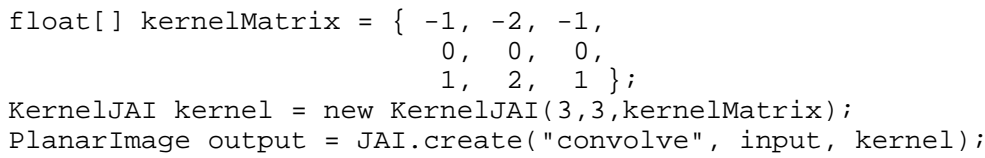

It is possible to use some operators to manipulate the whole bands in an image. For example, one can select some bands of a multiband image to create another image. The operator "bandselect" uses an input image and an array of integer band indexes to select bands from that image and add them in the specified order in the output image. The code in listing 9 shows how one can invert a RGB image by selecting the reverse order (BGR) of its bands. Usage of a ParameterBlock is not needed in this case.

Listing 9: Code for inverting a RGB image through band selection.

PlanarImage output = JAI.create ("bandselect", input, new int []$\{2,1,0\})$;

Another band manipulation operator is the "bandcombine" operator, which uses several image bands to combine them into a single multiband image. This method could be used to create a RGB image from three separate red, green and blue images, for example. The code snippet in listing 10 assumes that there is an array of image file names, read those images into an array of instances of P lanarImages using the "fileload" operator, then add those images to an instance of ParameterBlock (in the same order they were read). Finally, the "bandcombine" operator combine all images in the ParameterBlock and stores the result in a TIFF image through the "filestore" operator.

Listing 10: Code for creating a multiband image from several separated bands.

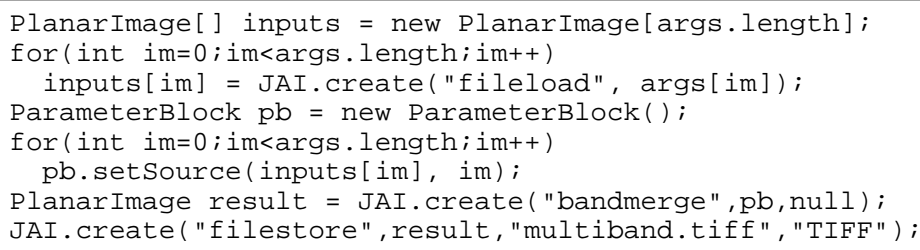

Some other simple operators are "add", "subtract", "multiply" and "divide", which performs basic arithmetic operations on two images, giving a third as result. The code snipped shown in listing 11 shows how two images (which are presumably already created or read from files) can be added, subtracted, multiplied or divided depending on which button on an user interface was clicked. 
Listing 11: Code for performing arithmetic operations on two images.

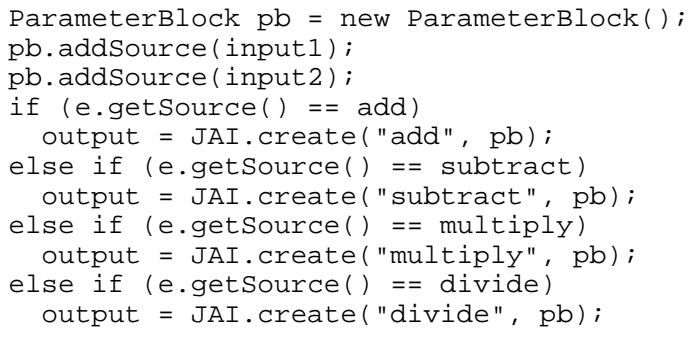

\section{Image data access}

Often there is the need to access individual pixel values from an image in order to perform some operation on that image - classification algorithms, for example, often require access to all pixels on an image for evaluation and classification.

One simple method that can be used to access pixels from an image is through the use of iterators. Iterators allow the access to the pixels on an image in a specific order. For example, an instance of Rect Iter scans the image column by column, from the top line to the bottom line, updating automatically the scanned pixels' coordinates and allowing the access (reading only) for all pixels in a band or for a pixel in a particular band the present scan coordinate. Another iterator, RandomIter allows the direct access to a pixel using user-specified $\mathrm{X}$ and $\mathrm{Y}$ coordinates.

Both iterators are actually interfaces, and opaque instances of classes that implement those interfaces can be obtained through factory methods in classes Rect IterFactory and RandomIterFactory, respectively. The factory methods require two arguments: one instance of PlanarImage (which will be the image being processed) and one instance of Rectangle, which will determine which rectangular subregion of the image will be considered for processing. If null is used instead of an instance of Rectangle, the whole image will be considered for processing.

The code snippet in listing 12 shows how one can dump all pixels' data values of an image to the console. The code gets the image dimensions, creates an array suitable for being filled with a pixel of the image (which may be a multiband image) and, iterating over all the pixels on the image, get its values and print them. Note that the method nextPixel must be called to increase the coordinates for the pixels.

Listing 12: Accessing all pixels in an image (using RectIter).

int width $=$ pi.getWidth ();

int height $=$ pi.getHeight (); 


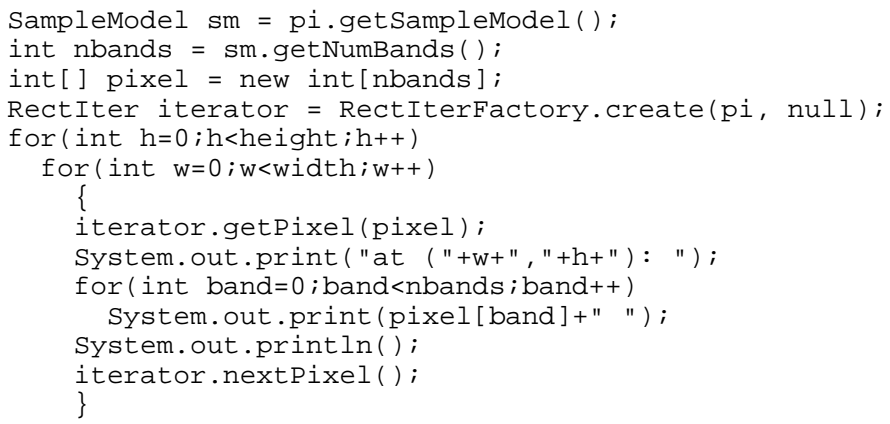

The code in listing 13 is similar to the one in listing 12, except that an instance of RandomIter is created and used, so when the method getPixel is called, one must provide $\mathrm{X}$ and $\mathrm{Y}$ coordinates to it.

Listing 13: Accessing all pixels in an image (using RandomIter).

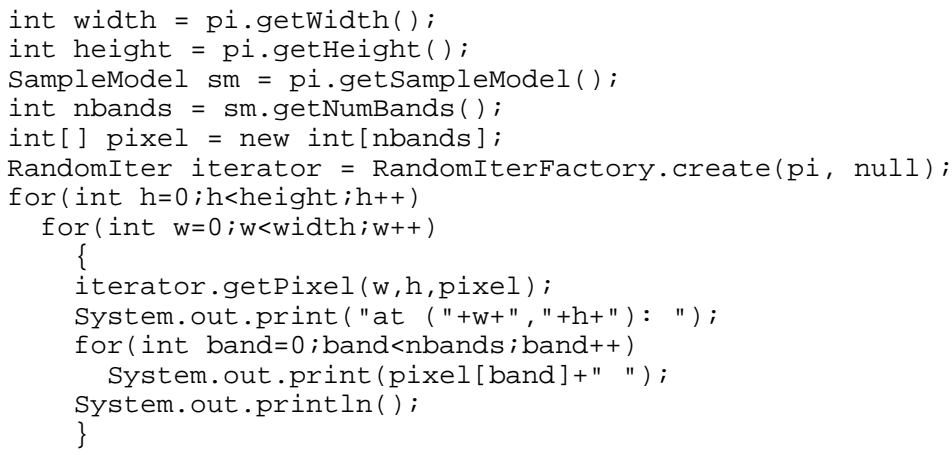

Although pixel data acessing with iterators is quite simple and straightforward, it causes some overhead on the performance of the applications, since, for each pixel, there must be some method calls (with image boundary verification). A faster pixel data acessing method is through the image raster.

As seen on section 2, the image pixels are stored in a Raster, which encapsulates both a DataBuffer and a SampleModel. The developer does not need to concern how the pixels are packed inside the Raster, its getPixel method and variants will get the pixels as a data array, while its get Sample method and variants will get a single data point (band of a pixel) from the image data. By getting a raster from the image and a data region from it, there will be fewer method calls and less overhead, so the application may perform 
better. On the other hand, since processing will be done by image chunks, more memory may be required, depending on the size of the region used for processing.

The code snipped in listing 14 shows how one can access all pixels in an image through the image's Raster. The code is similar to the shown in listings 12 and 13, except that an instance of Raster is created by calling the method getData on class PlanarImage, then the method getPixels of the instance of Raster is called to get all the pixels of the image in a suitable structure, which must have the required dimensions. The method getPixels get as parameters the coordinates of the upper left pixel location and its width and height, and a reference to the array which will get the data. In the example, the whole image was used as data source. It must be pointed that since the array which will get the data must be unidimensional, proper tracking of the pixels and band coordinates must be done.

Listing 14: Accessing all pixels in an image (using Raster.getPixels).

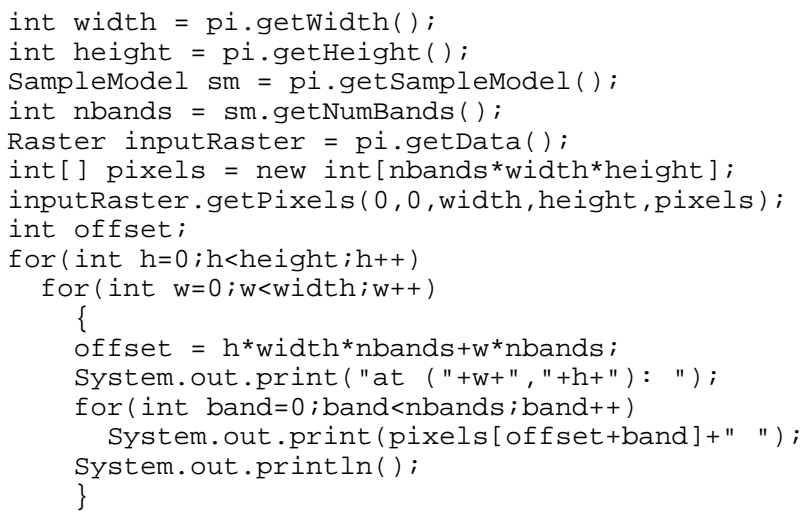

PlanarImages and Rasters are read-only, but it is easy to create an application that process the images' pixels and store them for further use. From the instance of Raster one can create an instance of WritableRaster with the same structure (but without the pixels' values) calling the method Raster. createCompatibleWritableRaster. The pixels' values can be obtained as shown in listing 14. After processing the pixels' values through the data array, the array can be stored again on the WritableRaster through its setPixels method, which arguments are the same as the used in Raster.getPixels.

A Raster or WritableRaster cannot be inserted again on a PlanarImage, but it is easy to create a TiledImage calling one of its constructors, which uses as parameters an instance of an already existing PlanarImage and the desired tiles width and height. The TiledImage will have the same dimensions and other features as the original 
PlanarImage and its setData method, with gets as an argument an instance of Raster or WritableRaster can be used to set its data. This TiledImage then can be further processed or stored.

Listing 15 shows the whole process. That listing shows a simple application where all pixels with values equal to zero are changed to 255 . The input to the code is a PlanarImage, and its output is a TiledImage with the original values changed.

Listing 15: Accessing all pixels in an image (for reading and writing).

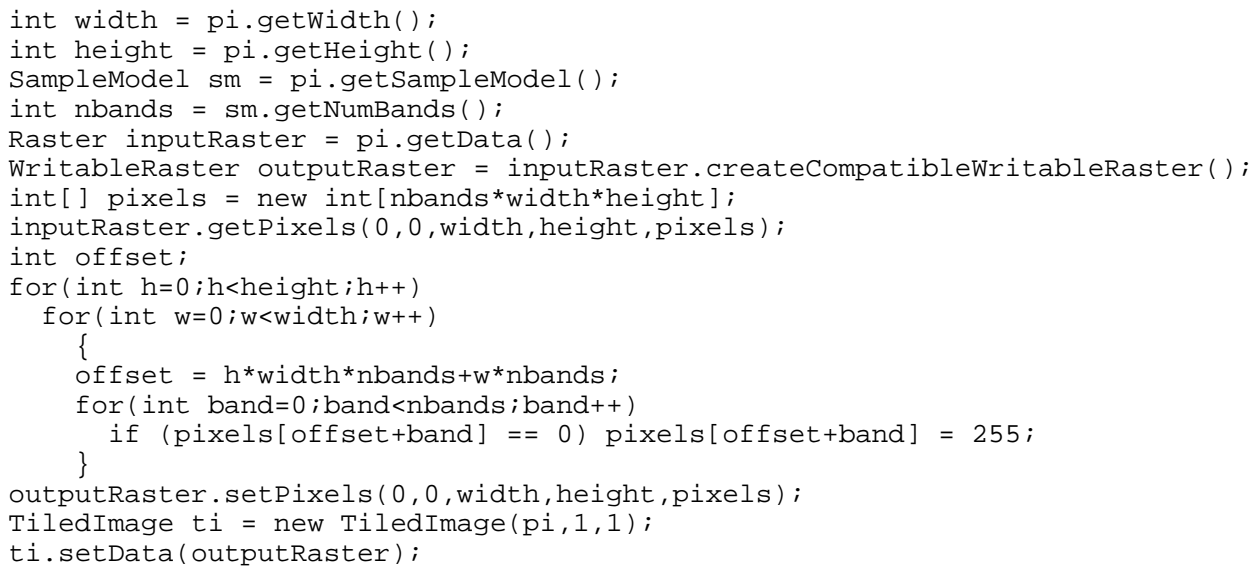

It is also possible to use writable iterators - for example, an instance of WritableRandomIter can be created through the method RandomIterFactory . createWritable and passing to this method an instance of TiledImage and an instance of Rectangle to set the bounds for the iterator or null to use the whole image. A writable iterator can be used in a similar way as a read-only iterator. The writable iterator will set the data directly on the output image, through its setPixel or set Sample methods.

\section{Simple visualization}

Visualization is an important step on an image processing application. Although it is possible to do read an image, process it and store the results on disk and use external applications to view those results, there are certain types of images which can be processed and stored but not easily viewed with generic applications - floating-point images and multiband images, for example. It may also more interesting to do the processing and visualization on a single Java application instead of relying on external applications. 
The JAI API provides a simple but extensible component for image display, implemented by the class DisplayJAI. This component inherits from JPanel and may be used as any other Java graphical component. This component can be used as-is or extended for different purposes.

One simple example is shown in listing 16. This complete Java application displays the image which file name is passed as a command line argument. An instance of DisplayJAI is created, using as argument for its constructor an instance of P lanar Image (the image on a DisplayJAI can be changed later through its set method). The instance of DisplayJAI is associated with a JScrollPane so images larger than the screen can be viewed through scrolling.

Listing 16: Simple usage of the DisplayJAI component.

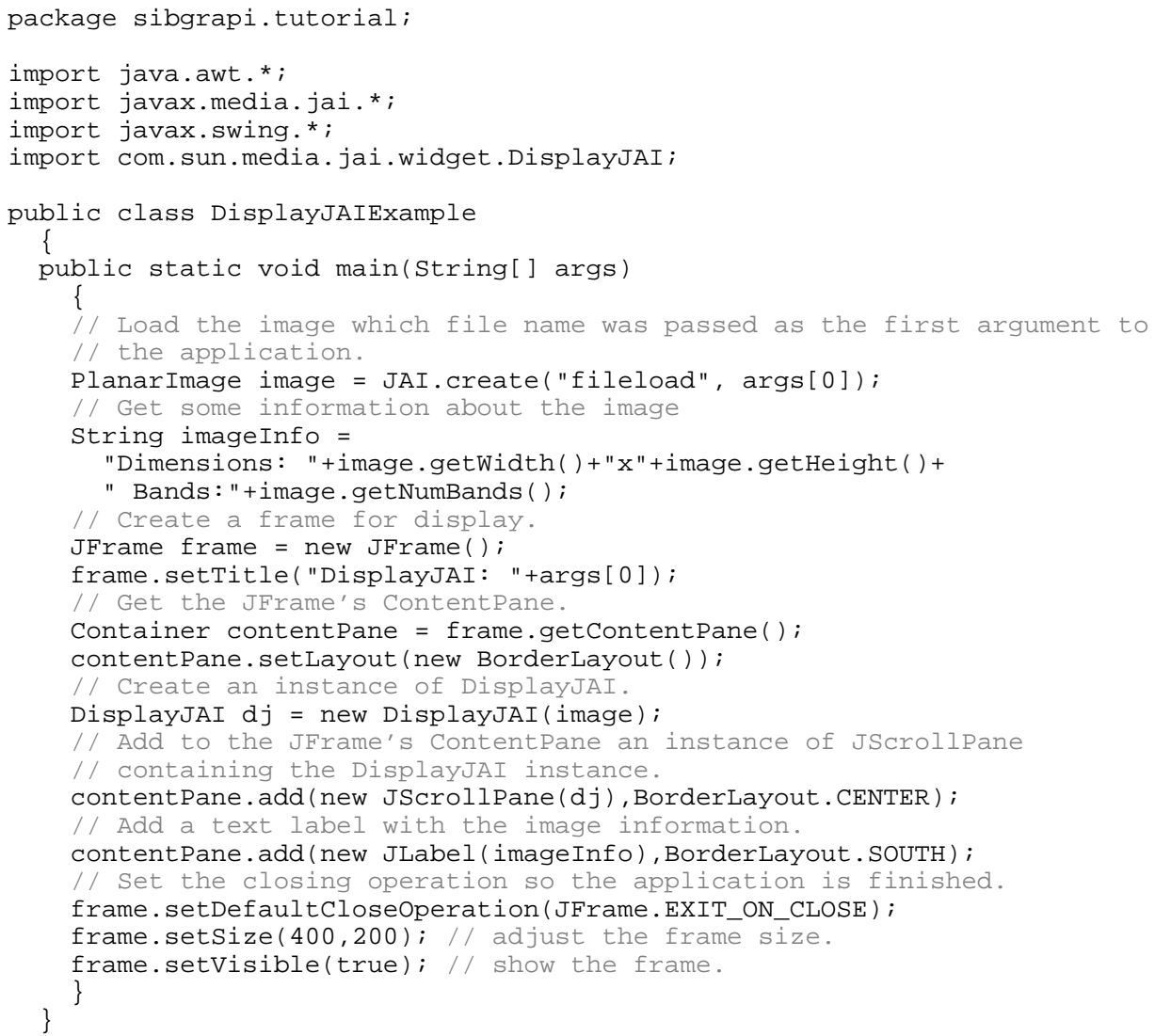


A screenshot of the application in listing 16 is shown in figure 3. The application assumes that the image can be displayed without problems, but will yield an exception if images with more than three bands are used.

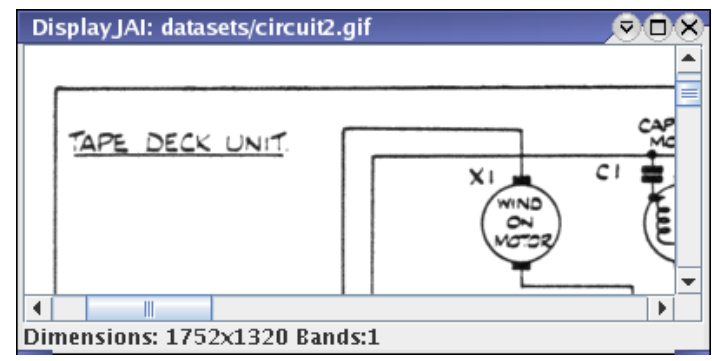

Figure 3. Screenshot of the DisplayJAI usage example application (listing 16).

\subsection{Visualization with a surrogate image}

The DisplayJAI component is able to display images which data type is not integer (e.g. floating point images) but its results are undefined - there is no explicit or controlable conversion of the image data. In this section, an example of extension of the DisplayJAI component will be shown. This example has two interesting points: it uses a surrogate image for display, which will be created from the original image data; and it also allows some basic user interaction, so the user can see the original value of the image pixel under the mouse cursor.

The component will be tailored for the displaying of digital elevation model (DEM) images, which are one-banded floating-point images, where the pixels are not a measure of a visible feature of the image but the elevation over the ocean level. In order to create a surrogate image which will visually represent the DEM, one must create a normalized and reformatted (casted) version of the original floating point image. The surrogate image pixels' values will be on the range $[0,255]$, normalized considering the minimum and maximum values of the DEM - in other words, all pixels on the surrogate image will be calculated as the value of the corresponding DEM pixel multiplied by $255 /(\max -\min )$ and added to $\min$, where $\max$ is the maximum DEM value and $\min$ the minimum DEM value. The surrogate image data type will also be set to byte.

In order to create the surrogate image with these rules, three JAI operators will be used. Those operators were not shown in section 3, therefore their description and usage will be presented now. The first operator is the "extrema" operator, which does not use any other parameter except for an input image. After this operator is applied, the user can call the method getProperty of the resulting Renderedop using "maximum" or "minimum" as 
arguments to get arrays of double values corresponding to the maximum and minimum pixel values per band. On this example, the DEM image is considered to have only one band.

The second operator that will be used in this example is the "rescale" operator, which uses as parameters (using a ParameterBlock) an input image, an array of double values for multiplication of the input image pixels and another array of double values for addition to the image pixels. If the dimension of those arrays is the same as the number of bands on the image, the multiplication and addition will be done on a per band basis, otherwise only the first value on the arrays will be used. The resulting image pixels are calculated as output $=$ input $\times m+a$ where $m$ and $a$ are the arrays for multiplication and addition, respectively.

The third operator used to create the surrogate image is the "format" operator, which get as parameters the input image and one of the constants TYPE_BYTE, TYPE_SHORT, TYPE_USHORT, TYPE_INT, TYPE_FLOAT or TYPE_DOUBLE, which are defined in the class DataBuffer. The resulting image data will be casted to the type corresponding to the DataBuffer constant.

Listing 17 shows the code for the modified component. This component (DisplayDEM) creates the surrogate image on its constructor, using the original image and the described steps for normalization and reformatting, also creating a RandomIter to obtain the original image pixels values. Part of the code on listing 17 allows the component to store the image data under the current mouse position, and to export those values as a StringBuffer.

Listing 17: Code for the DisplayDEM component.

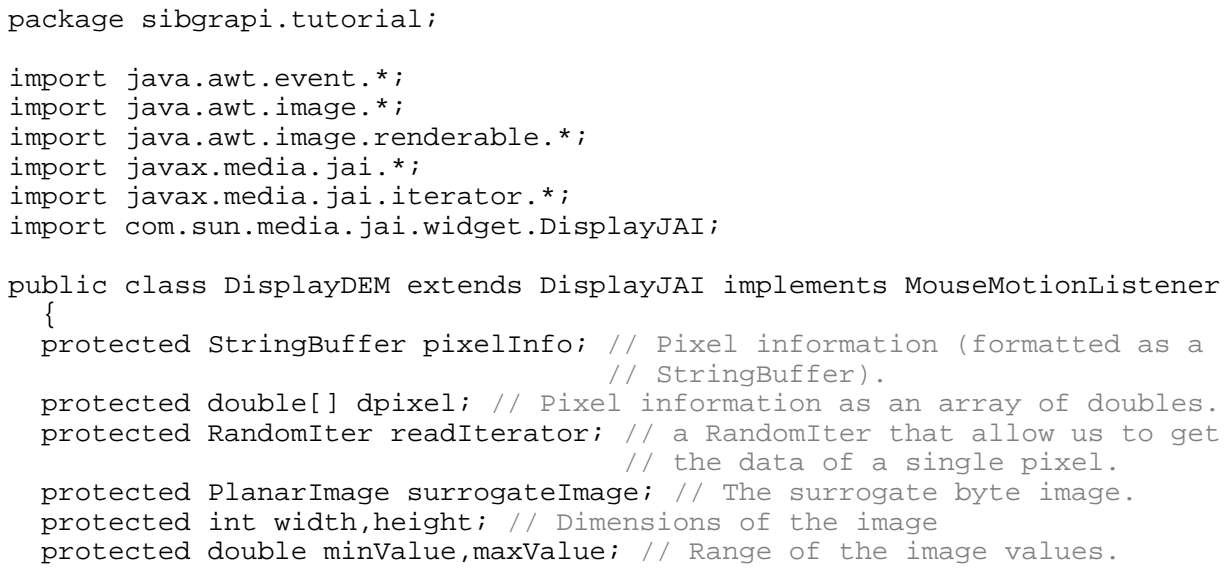




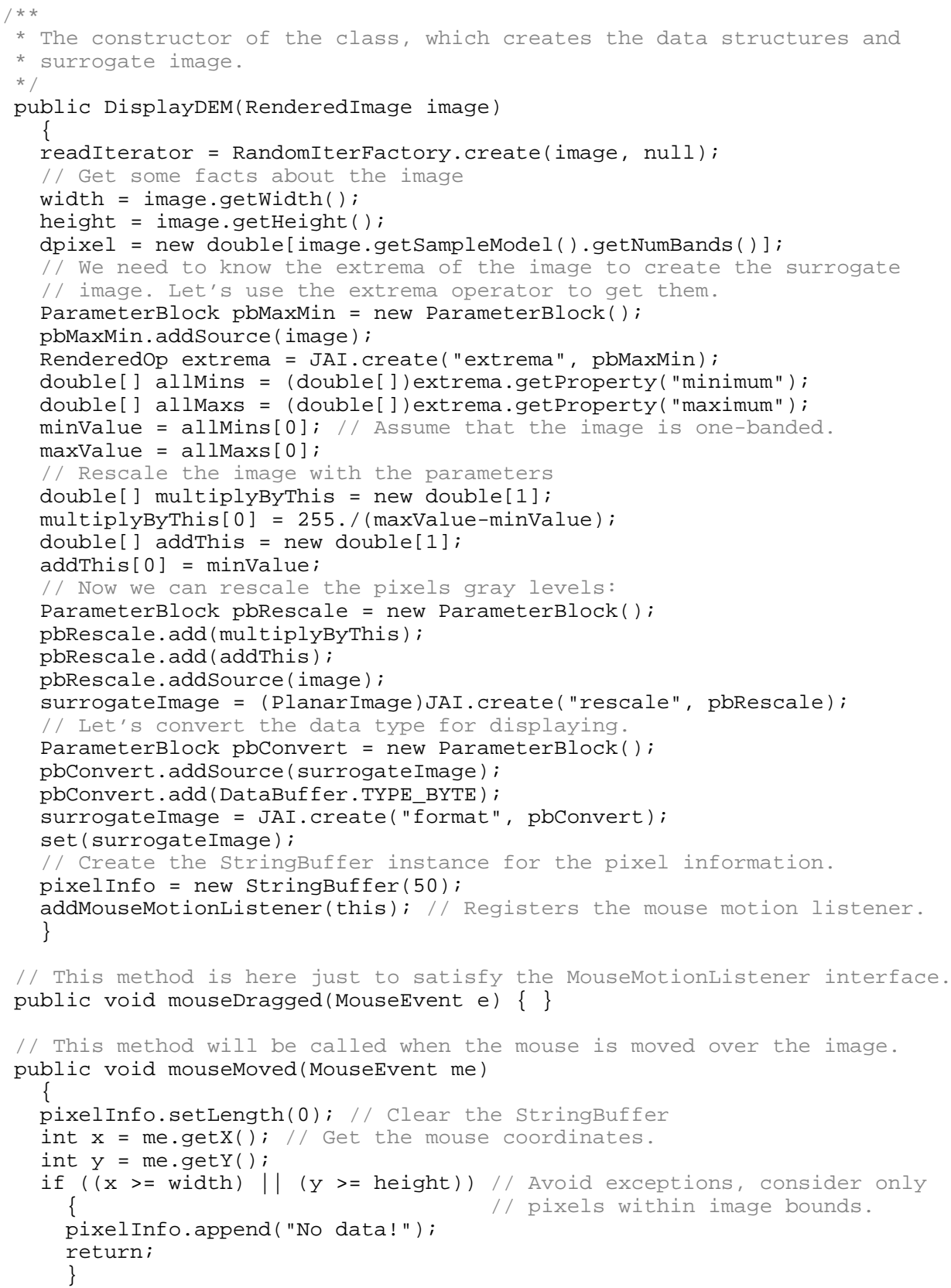




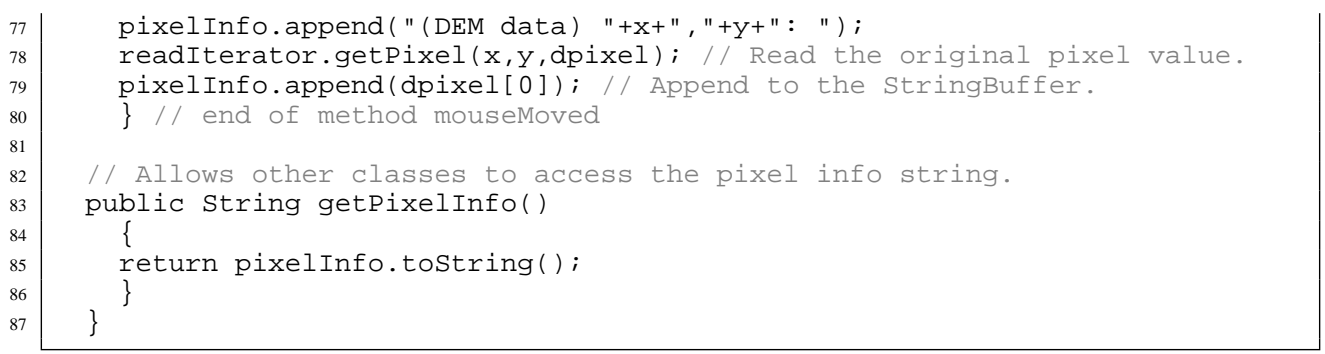

The section of code which uses the "extrema" operator is between lines 34 and 40 of the code in listing 17. Usage of the "rescale" operator is shown between lines 42 and 51, and usage of the "format" operator is between lines 53 and 56.

The DisplayDEM component can be used in any Java application with a graphical user interface. This application may or not use the original image pixel information that can be obtained through the DisplayDEM component. One example of application is shown in listing 18 - it is a simple application, which uses the component with a JLabel to show the original image value for the pixel under the mouse cursor.

Listing 18: Application which uses the DisplayDEM component.

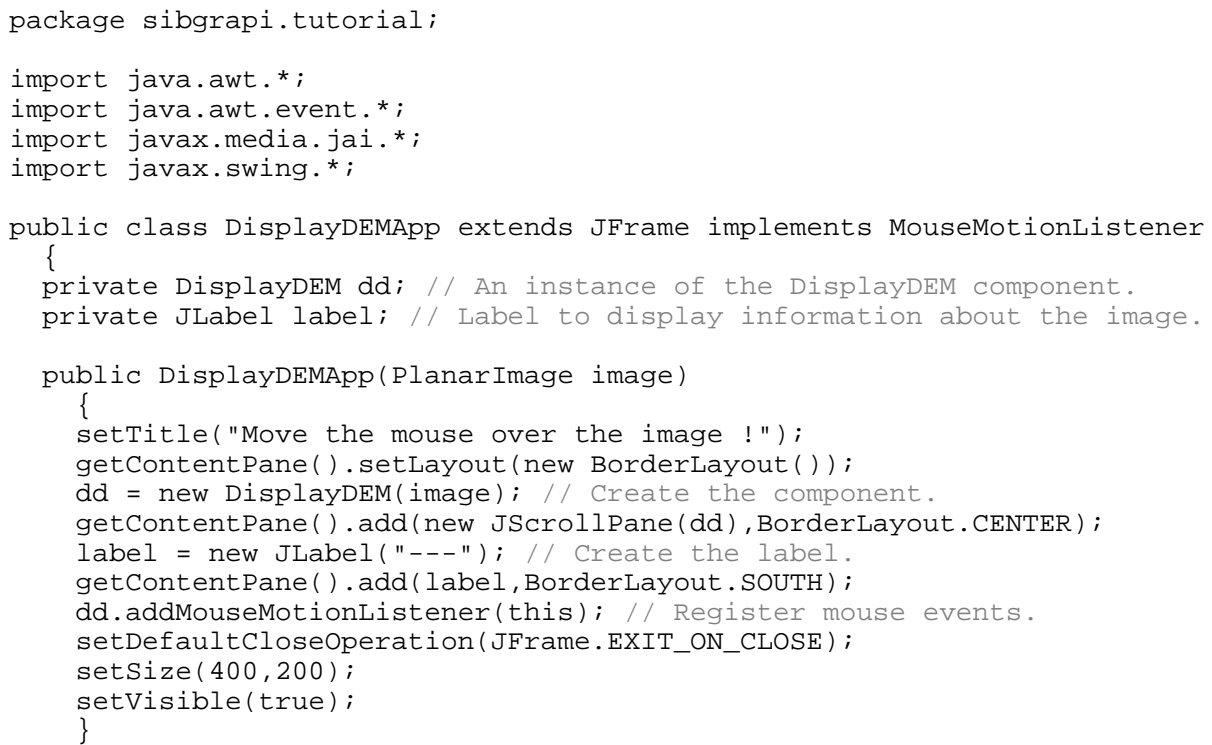




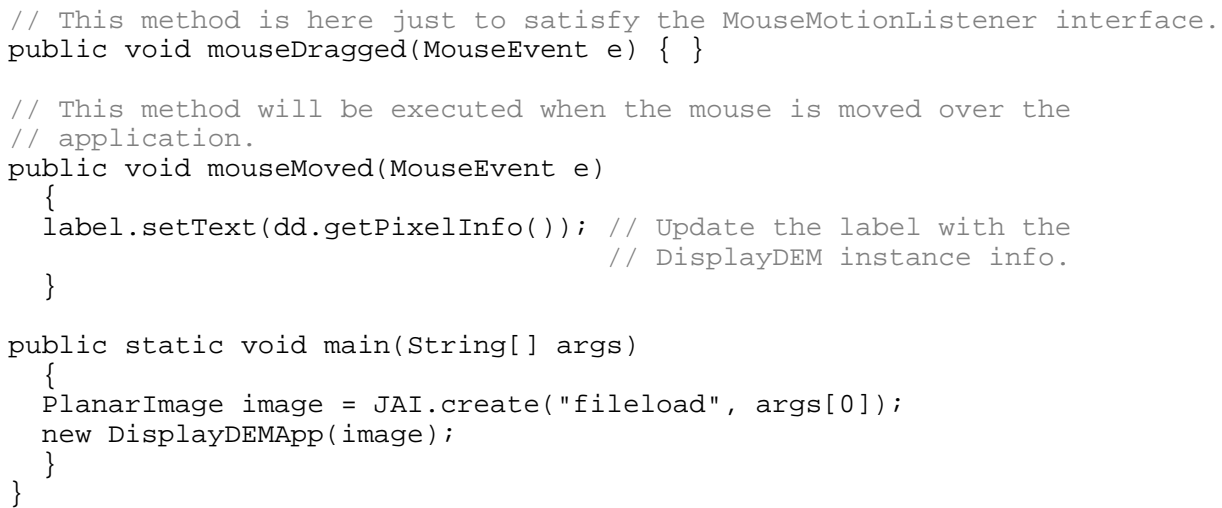

The figure 4 shows a screenshot of the DisplayDEMApp application (listing 18). The the bottom part of the application shows the image coordinates and original DEM value under the mouse cursor.

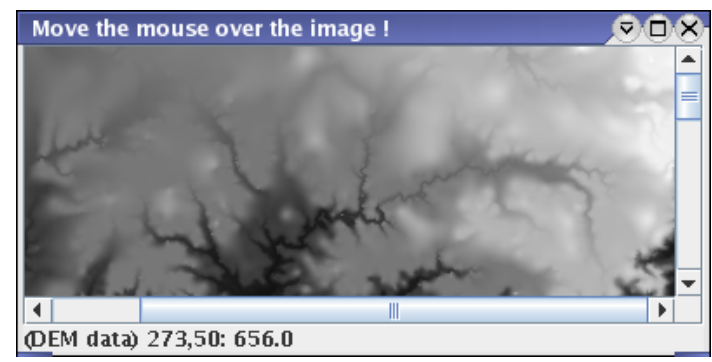

Figure 4. Screenshot of the DisplayDEMApp application (listing 18).

\subsection{Visualization of images with annotations}

Another frequent task in image processing applications is the display of an image with some kind of annotations over it - markers on the image, text, delimiters for regions of interest, etc. In this section a more complete set of classes will be described that allow the non-interactive creation of generic annotations and the display over images, using another extension of the DisplayJAI class.

In order to give a more complete and extensible example, an abstract class which encapsulates a drawable annotation is first devised. The class DrawableAnnotation is shown in listing 19, and simply declares an abstract paint method and a Color field, with a set and a get method for this field. Concrete classes that inherit from the Drawable- 
Annotation class must implement the paint method, which will draw the intended annotation using an instance of Graphics $2 \mathrm{D}$ as the drawing context.

Listing 19: Abstract class that encapsulates a drawable annotation.

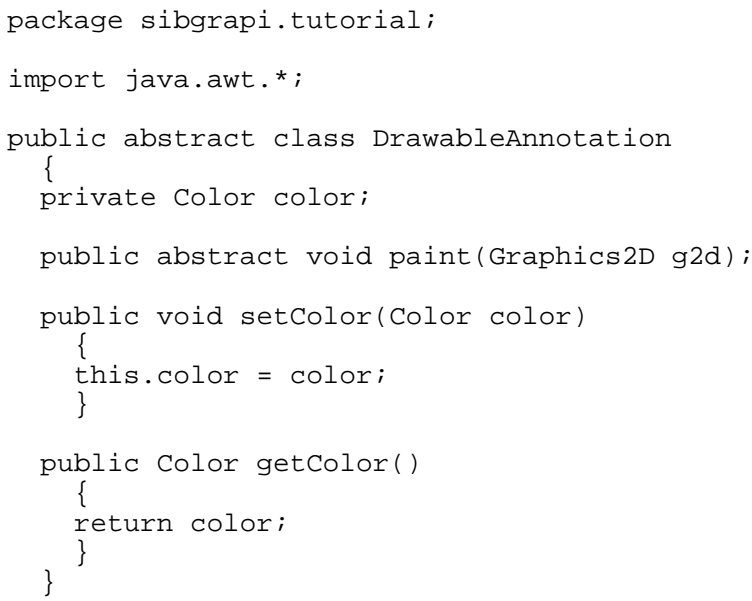

A concrete implementation of the drawable annotation class is shown in listing 20 . That class allows the drawing of a diamond-shaped annotation, using as parameters for its constructor a central point for the annotation and the diamond-shaped width and height in pixels and a pen width (to allow the creation of annotations which will be drawn with different pen widths).

Listing 20: Class that encapsulates a diamond-shaped annotation.

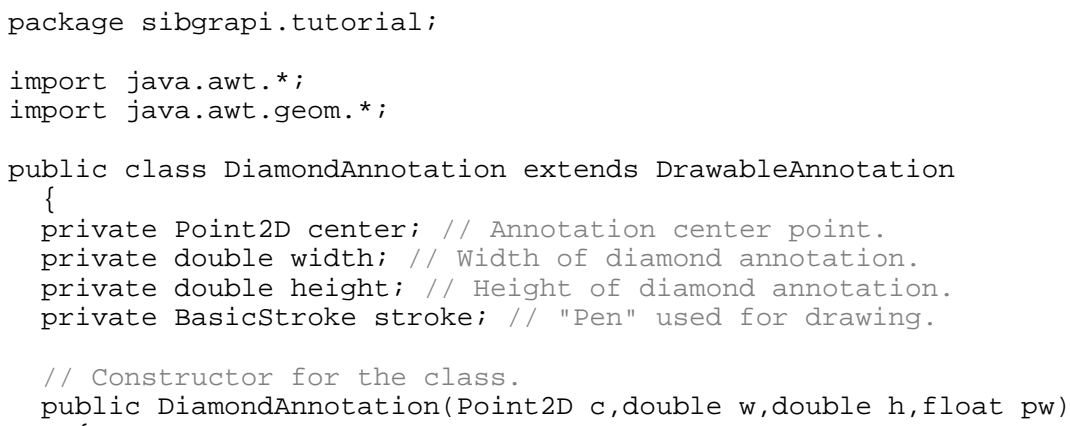




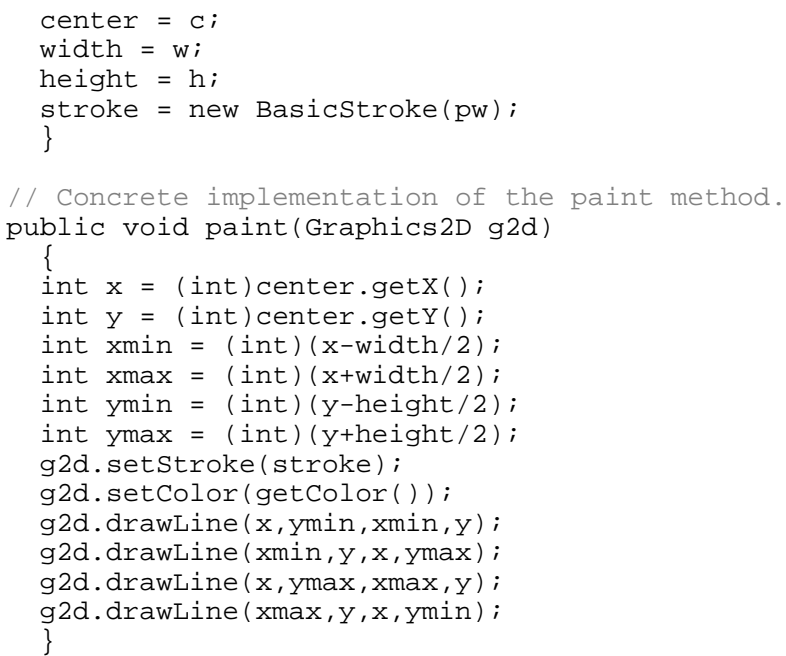

The main class in this section is the class that inherits from DisplayJAI and can display an image and draw annotations (instances of classes that inherit from DrawableAnnotation) over it. Annotations are stored as a list, and the class provides a method for adding annotations to the list.

This class overrides the paint method of the DisplayJAI class so after the image is painted (through a call to super.paint) all instances of annotations on the list have their paint method executed, using the same graphic context used to draw the image.

The code for the class that inherits from DisplayJAI (DisplayJAIWithAnnotations) is shown in listing 21.

Listing 21: Extension of the DisplayJAI class that draws annotations over the image.

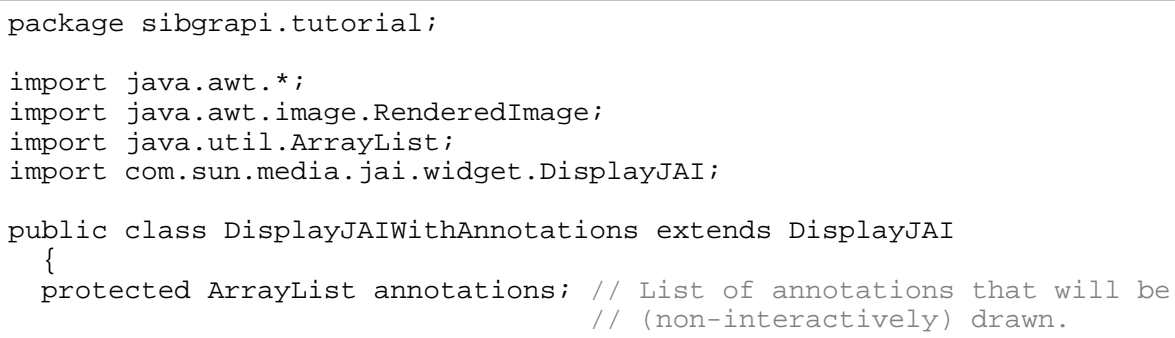




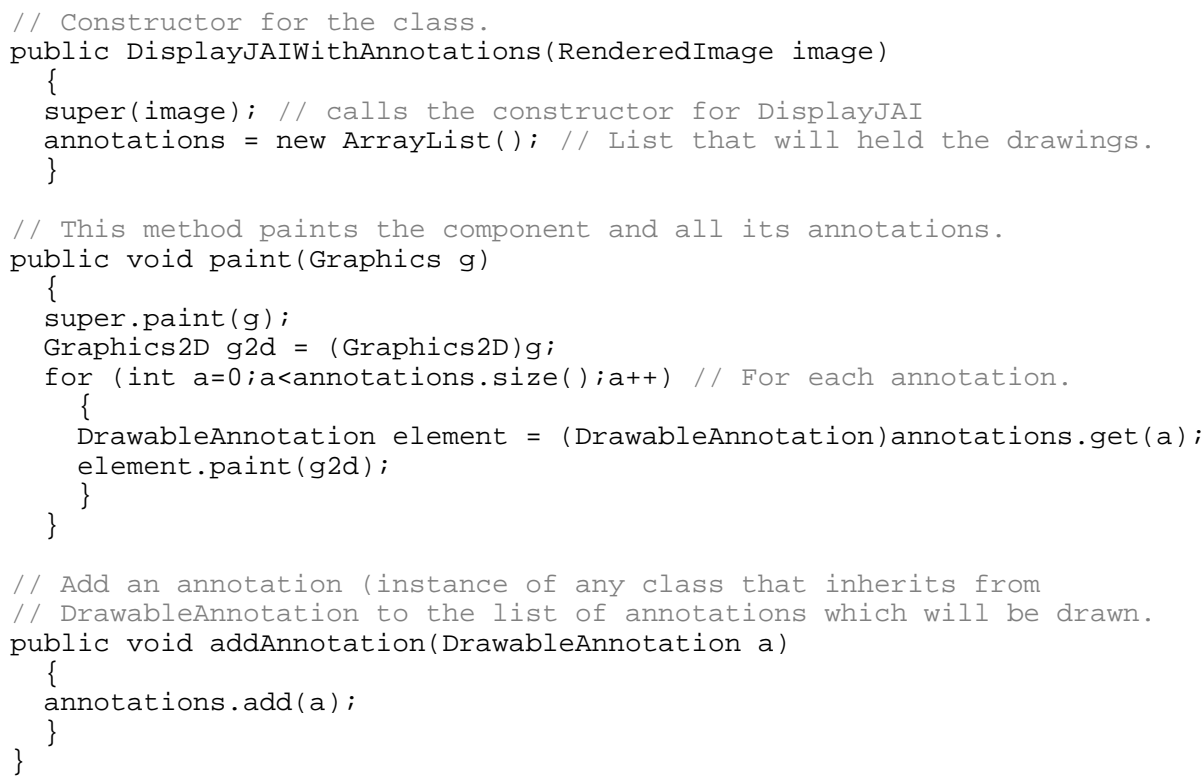

Finally, a Java application which uses the DisplayJAIWithAnnotations class is shown in listing 22. That application creates three instances of DiamondAnnotation and adds them to an instance of DisplayJAIWithAnnotations, which will be painted inside a JFrame.

Listing 22: Application which uses the DisplayJAIWithAnnotations component.

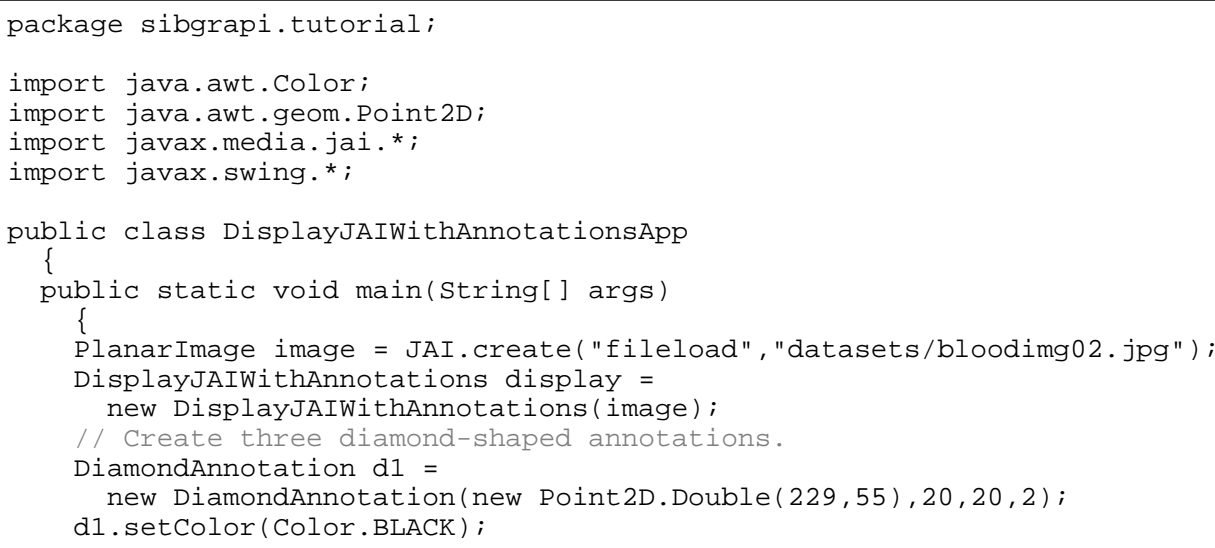




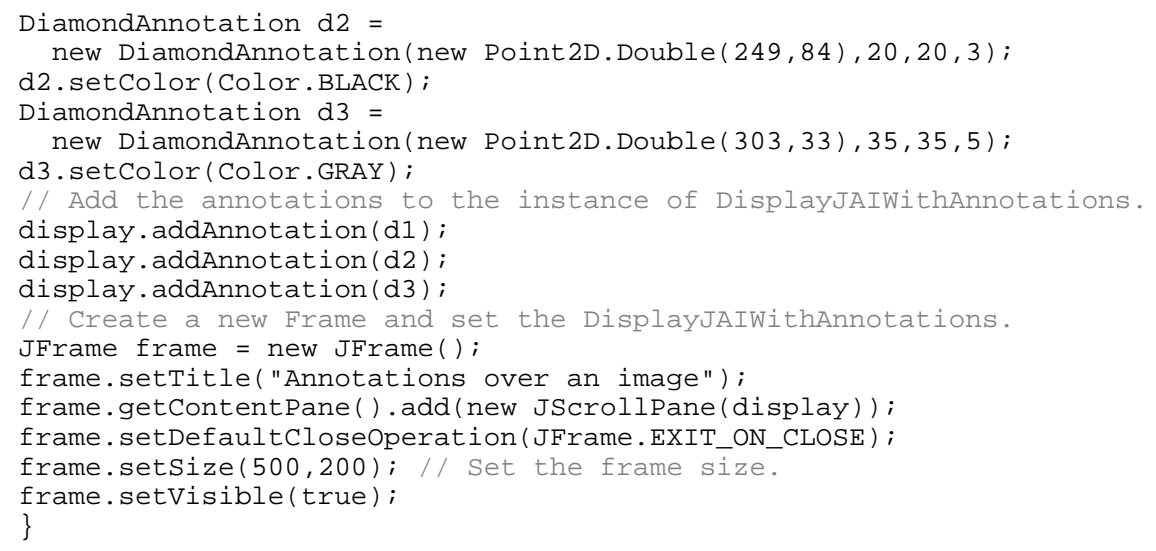

A screenshot of the DisplayJAIWithAnnotationsApp application (listing 22) is shown in figure 5 .

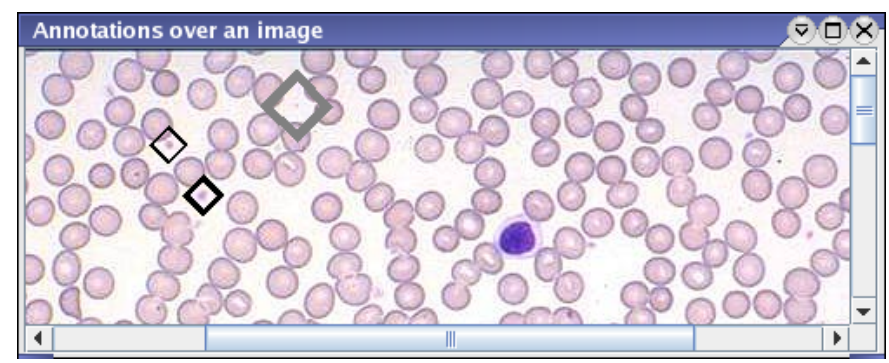

Figure 5. Screenshot of the DisplayJAIWithAnnotationsApp application (listing 22).

\section{Complete Example: A Fuzzy C-Means Implementation}

As a final example on this tutorial, let's see a complete implementation of the Fuzzy C-Means (FCM) clustering algorithm [7]. This algorithm iteractively cluster an image using fuzzy membership values instead of assigning each pixel to one and only one cluster.

The implementation is divided in two classes, one class which encapsulates the algorithm and which can perform the FCM in an image with any number of bands and another class which is an application that will call the methods in the first class. 
The first class is shown in listing 23. This class has a constructor to get information about the image and set the several data structure required for the execution of the FCM algorithm, a run method which executes the algorithm itself (calling several private helper methods) and a getRankedImage method which get a ranked clustering result. The use of the rank concept allows the user to get the best clustering result, i.e. in which each pixel is assigned to the cluster in which it had the largest membership function (using rank $=0$ ), or the second best, i.e. in which each pixel is assigned to the cluster in which it had the second largest membership function (using rank $=1$ ) and so on - this allows the exploration of clustering alternatives for cluster analysis.

Listing 23: The FuzzyCMeansClusteringTask class.

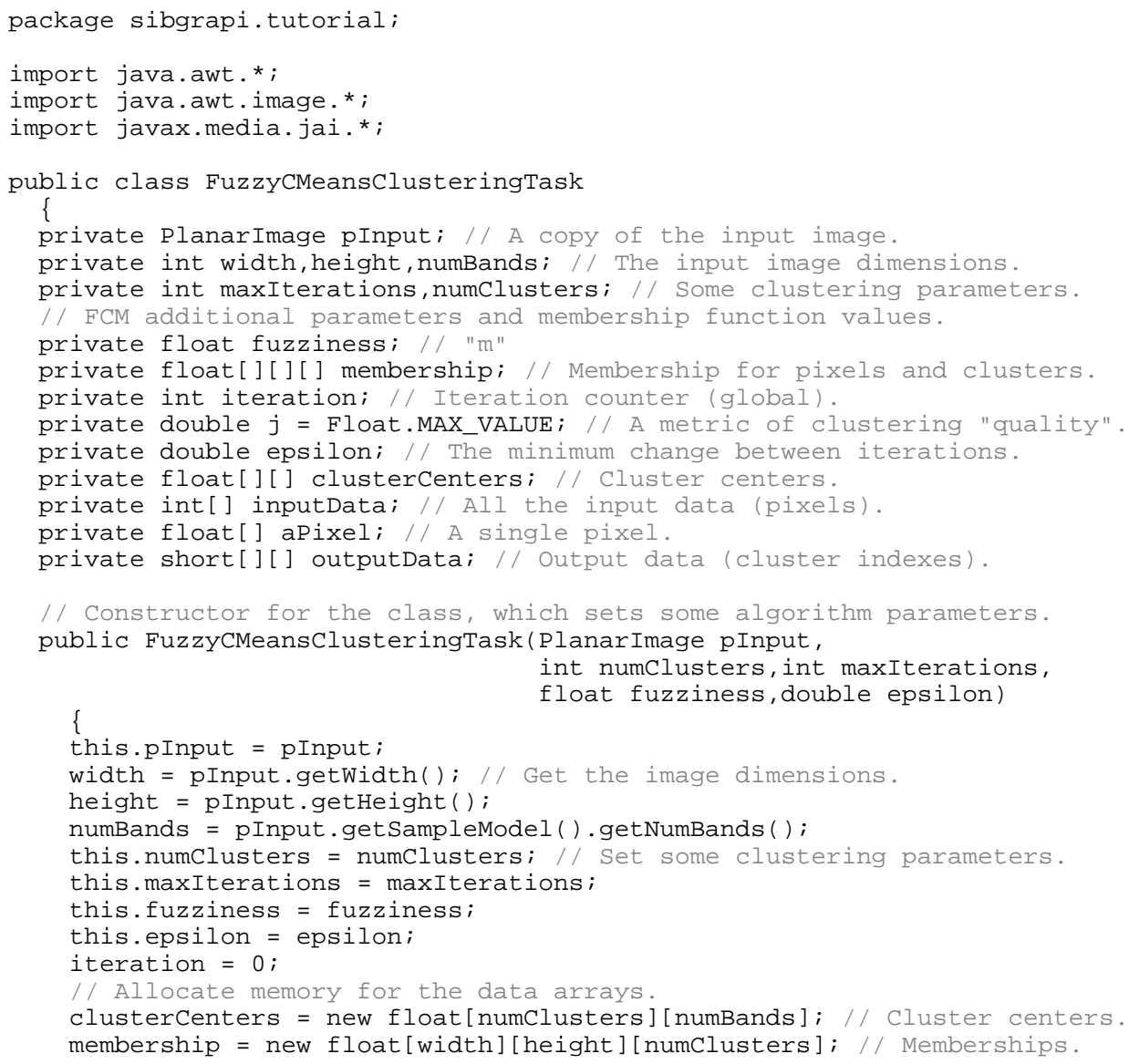


aPixel = new float [numBands]; // A single pixel.

outputData $=$ new short [width] [height]; // Cluster indexes.

// Gets the raster and all pixel values for the input image.

Raster raster $=$ pInput.getData ();

inputData $=$ new int [width*height*numBands];

raster.getPixels (0,0, width, height, inputData);

// Fill the membership function (MF) table with random values. The sum

// of memberships for a pixel will be 1, and no MF values will be zero.

for (int $h=0 ; h<$ height; $h++$ )

for (int $w=0 ; w<w i d t h ; w++)$

\{

float sum $=0 \mathrm{f}$;

for (int $\mathrm{C}=0 ; \mathrm{c}<$ numClusters; $\mathrm{C}++$ )

\{

membership $[\mathrm{w}][\mathrm{h}][\mathrm{c}]=0.01 \mathrm{f}+(\mathrm{fl}$ loat) Math.random();

sum $+=$ membership $[\mathrm{w}][\mathrm{h}][\mathrm{c}]$; \}

for (int $c=0 ; c<$ numClusters; $c++$ ) membership $[\mathrm{w}][\mathrm{h}][\mathrm{c}] /=$ sum;

\}

// This method performs the bulk of the processing. It runs the classic

// Fuzzy C-Means clustering algorithm:

// 1 - Calculate the cluster centers.

// 2 - Update the membership function.

// 3 - Calculate statistics and repeat from 1 if needed.

public void run()

\{

double lastJ; // The last "j" value (objective function).

lastJ = calculateObjectiveFunction(); // Calculate objective function.

// Do all required iterations (until the clustering converges)

for (iteration=0; iteration<maxIterations; iteration ++ )

\{

calculateclusterCentersFrommFs(); // Calculate cluster centers.

calculateMFsFromclusterCenters(); // Calculate MFs.

$j$ = calculateobjectiveFunction(); // Recalculate J.

if (Math.abs (lastJ-j) < epsilon) break; // Is it small enough?

last $\mathrm{J}=\mathrm{j}$;

\}

\}

// Calculates the cluster centers from the membership functions.

private void calculateclusterCentersFrommFs()

\{

float top,bottom; // Parts of the equation.

// For each band and cluster...

for (int $\mathrm{b}=0 ; \mathrm{b}<$ numBands; $\mathrm{b}++$ )

for (int $\mathrm{c}=0 ; \mathrm{c}<$ numClusters; $\mathrm{c}++$ )

\{

// For all data points calculate top and bottom parts of equation. top $=$ bottom $=0$;

for (int $h=0 ; h<$ height; $h++$ )

for (int $w=0 ; w<w i d t h ; w++$ )

\{

int index $=\left(h{ }^{*} w i d t h+w\right) * n u m B a n d s$;

top $+=$ Math.pow (membership [w] [h] [c], fuzziness)* 


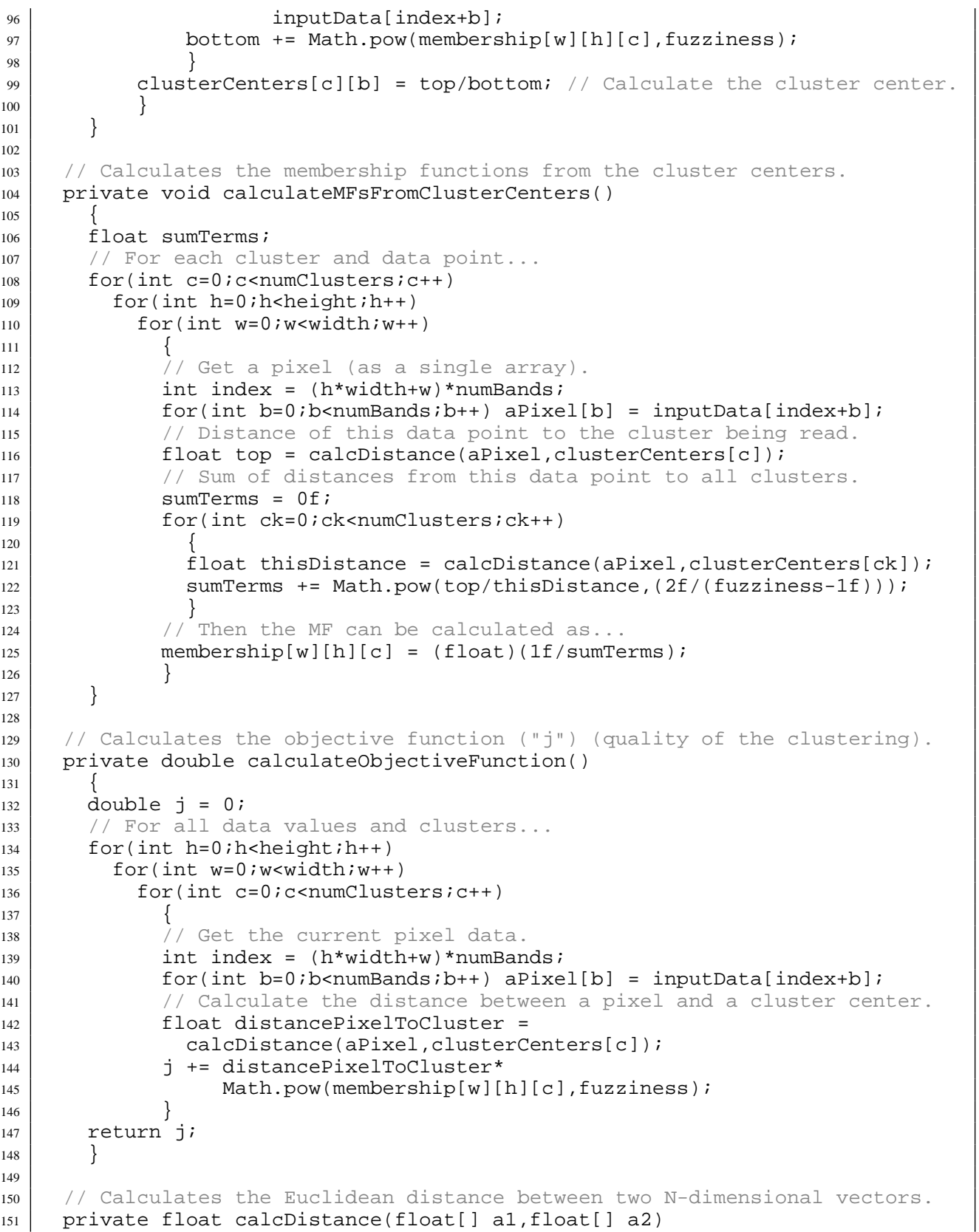




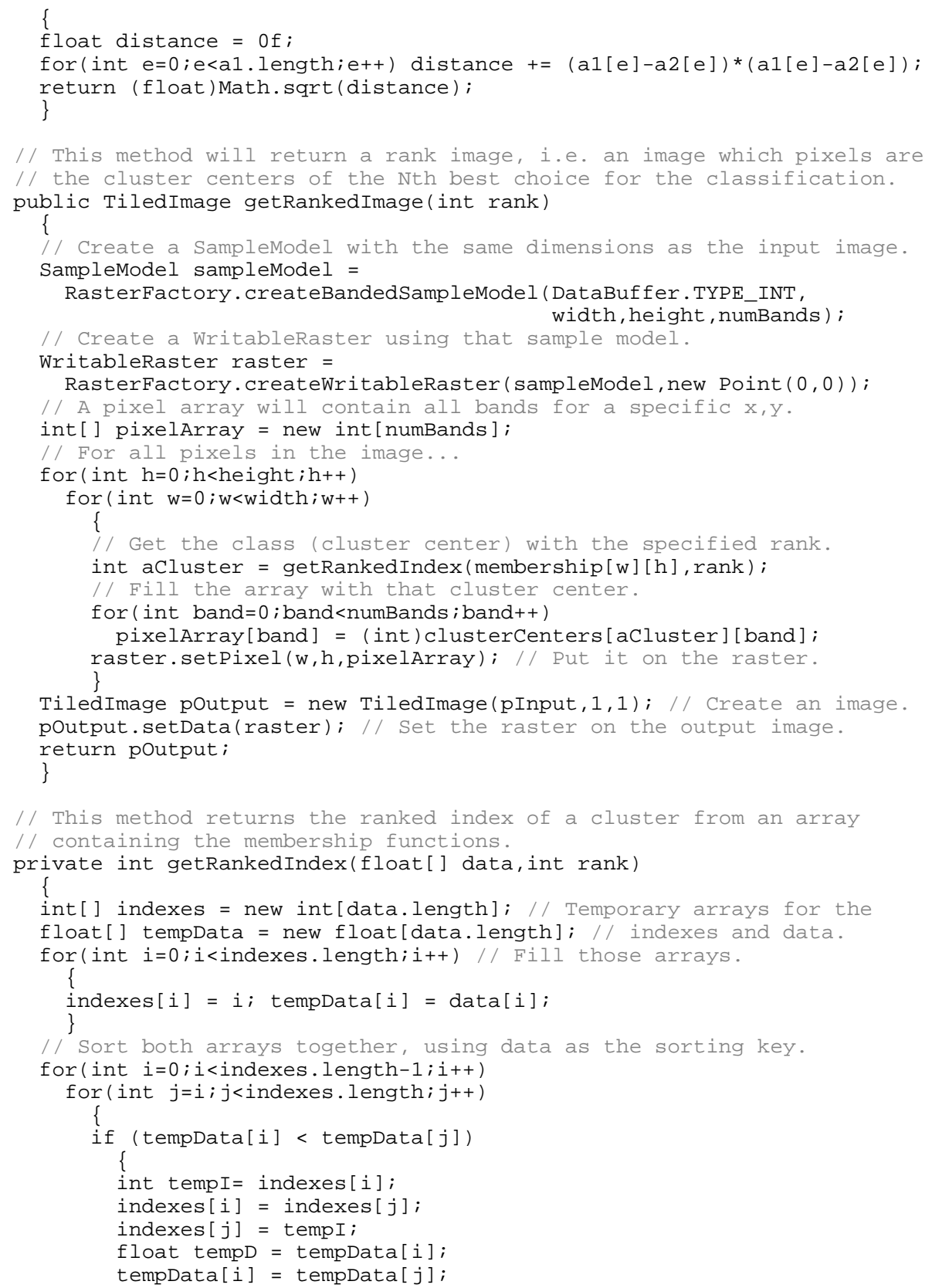




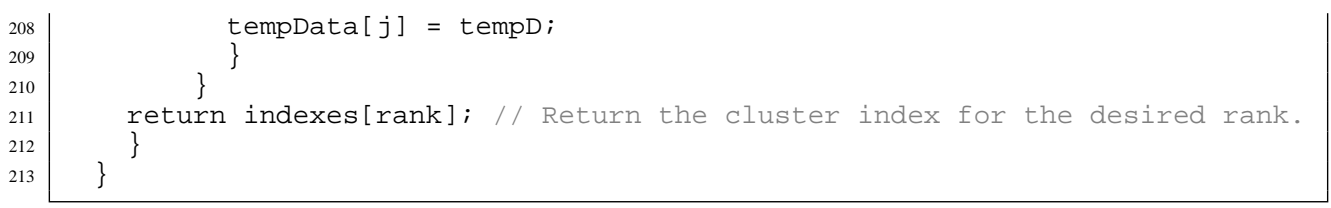

Listing 24 shows the FuzzyCMeansClusteringTaskApp class, an application which shows how the clustering methods on class FuzzyCMeansClusteringTask can be used to cluster an image which file name is passed as an argument to the application.

Listing 24: The FuzzyCMeansClusteringTaskApp class.

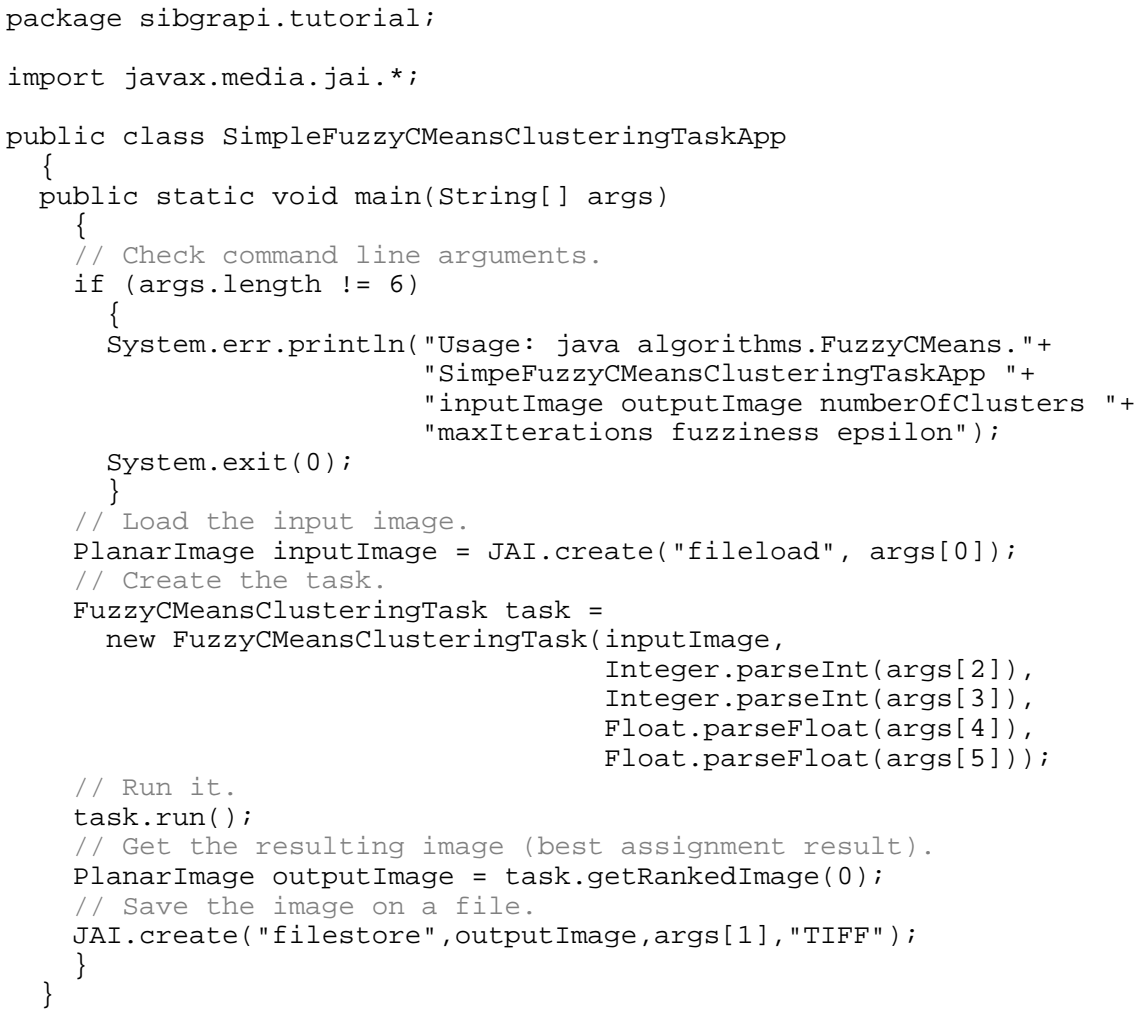




\section{Conclusions and further information}

Several examples of the Java Advanced Imaging API were shown in this tutorial. The author expects that the example, while simple and short, were enough to give the readers an idea of the workings of the JAI API and serve as basis for the development of more complex classes and applications.

Several listings shown in this tutorial are simplified versions of code found in [5], with comments removed in order to save space.

\section{References}

[1] Sun Microsystems, Java home page, http://java.sun.com (last visited in July 2004).

[2] Sun Microsystems, JAI (Java Advanced Imaging) home page, http://java.sun.com/products/java-media/jai/index.jsp (last visited in July 2004).

[3] Rodrigues, L.H. Building Imaging Applications with Java Technology, Addison-Wesley, 2001.

[4] Sun Microsystems, JAI (Java Advanced Imaging) Frequently Asked Questions, http://java.sun.com/products/java-media/jai/forDevelopers / jaifaq. html (last visited in July 2004).

[5] Santos, R. JAI Stuff (Tutorial): https : / jaistuff.dev.java. net (last visited in July 2004).

[6] Sun Microsystems, JAI (Java Advanced Imaging) API (Application Programming Interface) document home page, http://java.sun.com/ products/java-media/jai/forDevelopers/jai-apidocs/index.html (last visited in July 2004).

[7] Bezdek, J.C. Pattern Recognition with Fuzzy Objective Function Algorithms, Plenum Press, 1981. 